\title{
Das Erleben chronisch kranker Frauen in der Zeit von Schwangerschaft, Geburt und Wochenbett
}

\author{
Ute Lange, Friederike zu Sayn-Wittgenstein
}

Chronisch erkrankte Schwangere bewältigen die Herausforderungen des Mutterwerdens neben ihrer Therapie. Wie diese Frauen die Zeit von Schwangerschaft, Geburt und Wochenbett subjektiv erleben und bewältigen, war Thema einer Analyse qualitativer Studien.

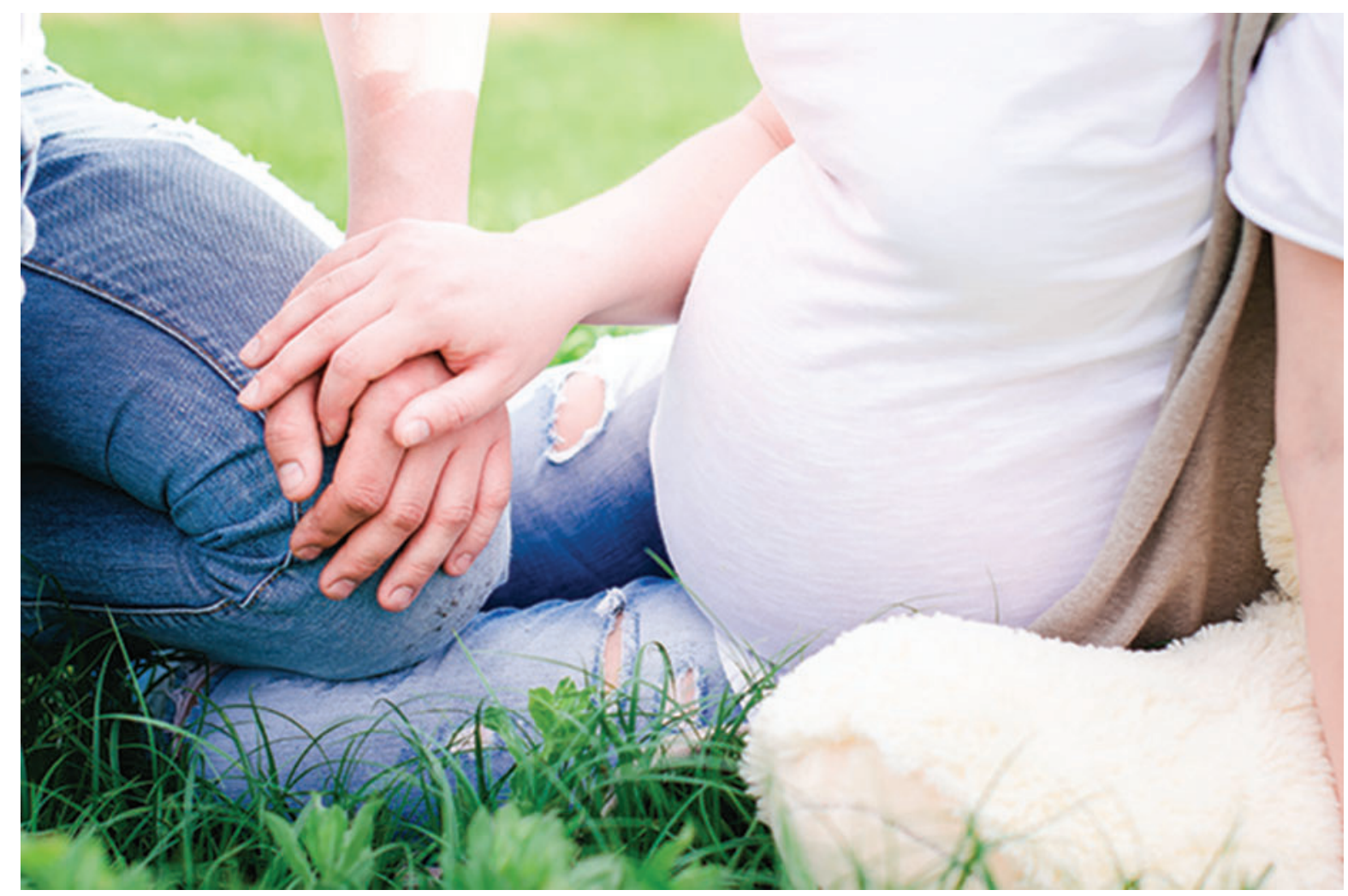

Abb. 1 In der Zeit rund um Schwangerschaft, Geburt und Wochenbett ist für chronisch kranke Frauen der Rat von Hebammen weitaus bedeutsamer als der des sozialen Umfelds. (Foto: romaxa86 - stock.adobe.com - Symbolbild)

\section{Hintergrund}

Angesichts der wachsenden Zahl chronisch erkrankter Menschen auch in der reproduktiven Lebensphase gewinnt das Thema der Versorgung betroffener Frauen in Schwangerschaft, Geburt und Wochenbett an Aktualität [1] [2]. Frauen mit chronischen Erkrankungen müssen in der Zeit rund ums Mutterwerden sowohl die grundsätzlichen Herausforderungen dieser Lebensphase als auch die Besonderheiten ihrer Erkrankung bewältigen. Beide Themenkomplexe erfordern ein hohes Maß an Aufmerksamkeit, binden Zeit und Energie [3]. Allein eine chronische Erkrankung ist durchgehend mit einer Krankheits-, Biografie- und Alltagsarbeit verbunden [4]. Die Komplexität der Fragestellungen, die sich aus dem 
Spannungsfeld von Erkrankung und Mutterwerden ergibt, kann bei den betroffenen Frauen zu einem allgemein höheren Level an Überforderung, Ängsten, Sorgen und Ambivalenzen führen [5]. Auch sind sie einer erhöhten Komplexität von Entscheidungsprozessen mit teils widersprüchlichen Anforderungen ausgesetzt [3]. Bekannt ist, dass sich die Erfahrungen rund um die Geburt bei Frauen mit chronischer Erkrankung in besonderer Weise auf das längerfristige reproduktive Verhalten wie einen weiteren Kinderwunsch und das Gelingen der Familienbildung auswirken [6]. Damit stellt sich auch die Frage, wie chronisch erkrankte Frauen im Gesundheitssystem unterstützt und entlastet werden können. U.a. weist das Wissen darum, dass langfristiger Stress zu Schwangerschaftskomplikationen führen kann, auf die Bedeutung solcher Fragstellungen hin [7] [8].

Die Berücksichtigung sowohl medizinischer als auch psychosozialer Faktoren und der Bedürfnisse betroffener Frauen ist eine Voraussetzung für die Entwicklung angepasster Versorgungskonzepte rund um die Geburt. Diese sollten sowohl eine Minimierung negativer gesundheitlicher Folgen für Mutter und Kind als auch das Gelingen der Familienbildung zum Ziel haben. In einem ersten Schritt ist dazu das Verstehen der Sichtweisen Betroffener notwendig. Vor diesem Hintergrund werden in dieser Literaturanalyse Studien untersucht, die das Erleben von Frauen mit chronischer Erkrankung in der Zeit von Schwangerschaft, Geburt und Wochenbett fokussieren. Es wird der Frage nachgegangen, welche Konsequenzen sich aus den Ergebnissen für die weitere Forschung und die professionelle Betreuung insbesondere durch Ärzte bzw. Ärztinnen und Hebammen ergeben. 11 Studien bilden die Grundlage für diese Literaturanalyse. Sie umspannen den Zeitraum von 1987 bis 2014, 8 der Studien wurden ab dem Jahr 2000 veröffentlicht. 5 von ihnen kommen aus Großbritannien, 3 aus den USA und jeweils eine aus Neuseeland, Irland und Schweden.

\section{Chronische Erkrankungen bei Frauen in der reproduktiven Lebensphase}

Chronische Erkrankungen zeichnen sich nach Definition der WHO durch Dauerhaftigkeit, nicht Heilbarkeit und eine erhöhte Inanspruchnahme des Gesundheitssystems aus [1] [2]. Grundsätzlich steigt die Zahl betroffener Menschen in den Industrienationen an, was unter anderem auf weitgehend verfügbare Diagnose- und Therapiemöglichkeiten und damit eine verlängerte Lebenserwartung zurückgeführt werden kann [9]. In Deutschland wird nicht zentral erfasst, wie hoch der Anteil chronisch erkrankter Frauen in der Gruppe der Schwangeren ist. Einen Hinweis gibt die Studie von Kersten et al. [12], die Daten hinsichtlich der Prävalenz chronischer Erkrankungen und des Geburtsoutcomes untersuchte: $21,4 \%$ der Schwangeren $(n=1141)$ wurden als chronisch krank identifiziert, am häufigsten traten bei ihnen Allergien (11,3\%), Asthma Bronchiale (2,7\%) und Schilddrüsenerkrankungen (2,3\%) auf. Weiterhin zeigten Hauterkrankungen (2,2\%), Hypertonien $(1,1 \%)$ und Migräne $(1,5 \%)$ hohe Prävalenzen. Jede fünfte schwangere Frau im Studiengebiet leidet demnach an mindestens einer chronischen Erkrankung. In der Bundesauswertung des Instituts für Angewandte Qualitätsförderung und Forschung im Gesundheitswesen (AQUA) [13] fällt auf, dass der Anteil der Schwangeren mit einer möglichen chronischen Erkrankung in fast allen erfassten Parametern kontinuierlich ansteigt.

\section{STICHWORT}

\section{Chronizität}

Chronizität wird in dieser Literaturübersicht als ein übergeordnetes Konzept angesehen und dabei vorausgesetzt, dass sich einzelne Aspekte des Erlebens der Frauen trotz der unterschiedlichen Erkrankungen generalisieren lassen. In 7 Studien setzt sich die Untersuchungsgruppe aus Frauen mit verschiedenen Erkrankungen wie Diabetes mellitus, Lupus Erythematodes, Herzerkrankungen, Epilepsie und Multipler Sklerose zusammen. In 4 weiteren werden die Erfahrungen von Frauen mit jeweils einer Erkrankung erfasst, nämlich Multiple Sklerose, Diabetes mellitus oder Rückenmarksverletzungen.

\section{Schwangerschaft im Spannungsfeld von Freude und Belastung}

Frauen mit einer chronischen Erkrankung stehen größeren Herausforderungen in der Schwangerschaft gegenüber als die Mehrheit der gesunden Frauen. In den meisten Studien wird jedoch deutlich, dass die Freude auf das Kind und die Chance auf eine Mutterschaft als vorrangig erlebt werden und andere teils schwierige Erfahrungen ins Positive wenden [32] [33] [34]. Trotzdem müssen die von Tyer-Viola und Lopez [33] als „blessings and burdens" benannten Herausforderungen, also Segen und Belastungen einer Schwangerschaft, von den Frauen parallel bewältigt werden, was auch die Wahrscheinlichkeit für psychosoziale Probleme erhöht. Die Komplexität der Anforderungen führt zu der Forderung Betroffener, das Thema der Mutterschaft mit chronischer Erkrankung nicht vornehmlich aus medizinischer Perspektive, sondern es umfassender zu betrachten [32] [33] [34].

Einige Studien identifizieren den Wunsch nach Normalität als ein tragendes Bedürfnis der befragten Schwangeren [33] [34]. Diesem Wunsch steht die Realität mit der durchgängigen Möglichkeit medizinischer Komplikationen gegenüber. Als Belastung wird von den Befragten 
wahrgenommen, dass viele medizinische Maßnahmen mit Einschränkungen des Privatlebens verbunden sind. Es werden spezifische Strategien beschrieben, um mit diesem Spannungsverhältnis von persönlichen Bedürfnissen und notwendigen Therapien und Diagnosen umgehen zu können. Dies sind insbesondere die des Abwägens, Ausbalancierens und Kontrollierens. Der Erfolg dieser Strategien hängt von dem Zugang zu Informationen, dem Grad an Unterstützung, der Verfügbarkeit angepasster Therapien und der persönlichen Kontrollüberzeugung der Frauen ab [2].

Die Art der Erkrankung bedingt verschiedene Problemlagen. So wird das fürsorgliche Verhalten des sozialen Umfelds sowie der professionellen Akteure des Gesundheitssystems bei nach außen sichtbaren bzw. nicht sichtbaren Erkrankungen unterschiedlich wahrgenommen. Frauen mit sichtbaren Erkrankungen erleben mehr Hilfsangebote als solche mit Erkrankungen, die für andere nicht wahrnehmbar sind. Sie sind aber auch negativen und unverständlichen Reaktionen anderer bezüglich ihres Schwangerseins oder Mutterseins am stärksten ausgesetzt [32] [35]. Diabetes hingegen als nicht sichtbare Erkrankung löst weniger Reaktionen hervor, bedarf aber einer ständigen Überwachung und hat daher wesentlichen Einfluss auf den Alltag betroffener Frauen. Für diese ist damit der Verlust der Kontrolle über die Lebensführung dominant. Berg [36] fasst das mit „to master or to be enslaved" zusammen.

\section{Bedeutung des sozialen Umfelds}

Einige interviewte Frauen machen die Erfahrung, dass der Entschluss zu einem Kind sowohl in ihrem sozialen Umfeld, wie dem Partner, Freunden und Arbeitskollegen, als auch bei den betreuenden Ärzten bzw. Ärztinnen Skepsis auslöst und sie sich für ihren Kinderwunsch rechtfertigen müssen. Obwohl diese Skepsis belastend ist, räumen die Frauen oftmals Verständnis für die Vorbehalte ein. Ihrer Einschätzung nach beruhen sie insbesondere auf der Sorge, als Erkrankte könnten sie den körperlichen Belastungen der Schwangerschaft und dem Leben mit einem Kind nur eingeschränkt gewachsen sein oder auch langfristige gesundheitliche Schäden davontragen [35]. Andererseits wird von den betroffenen Frauen aber auch vermutet, dass es allgemeine Vorbehalte gegen eine Mutterschaft bei chronischer Erkrankung geben könnte und sie dem allgemeinen, gesellschaftlich akzeptierten Mutterbild nicht entsprechen [29] [34].

Ein wesentlicher Anteil der Ängste von Partner und Familie sind nach Ansicht der befragten Frauen auch darin begründet, dass diese mehr Aufgaben in der Bewältigung des Alltags übernehmen müssen als allgemein üblich und somit von vorne herein belasteter sind [37] [38]. Einige Frauen äußern daher den Wunsch, dass
Betreuungskonzepte auch auf die Gruppe der begleitenden Personen ausgeweitet und deren besondere Situation berücksichtigt werden sollte. Solche Konzepte würden ihnen und ihren Vertrauten mittelbar Entlastung verschaffen [29] [34]. Corbin [4] betont, dass sich ein unterstützendes soziales System auf das positive Erleben und einen komplikationsarmen Verlauf der Schwangerschaft auswirkt. Die oftmals aufwändigen medizinischen Maßnahmen erfordern grundsätzlich viel Zeit und Energie. Aufgrund dieser oftmals angespannten Lage können die negativen Effekte zusätzlicher Belastungen wie familiärem Stress auf das Risiko von Schwangerschaftskomplikationen oder dem Gefühl von Überforderung in besonderem Maße auswirken [2].

Oftmals versuchen die befragten Frauen, in der Zeit rund um die Geburt eines Kindes Kontakt zu Betroffenen in ähnlichen Lebenssituationen aufzunehmen, was meist schwer zu realisieren ist [28]. Dies bestärkt sie in der Wahrnehmung, anders als andere Schwangere zu sein und eine Außenseiterrolle einzunehmen [37].

\section{Schwangerschaft, Therapie und medizinische Überwachung}

Lipson und Rogers (2000) stellen die These auf, dass die Erfahrung des Mutterwerdens bei Frauen mit chronischer Erkrankung wesentlich von dem jeweiligen Gesundheitssystem beeinflusst wird [35]. Auch gesunde Schwangere werden in den Industrienationen intensiv medizinisch betreut, trotzdem unterscheidet sich ihre Situation wesentlich von der chronisch Erkrankter. Frauen mit chronischer Erkrankung sind bereits vor der Schwangerschaft in kontinuierlicher Behandlung von Ärzten bzw. Ärztinnen und anderen Vertretern bzw. Vertreterinnen des Gesundheitssystems. Oftmals ist das Schwanger werden bei ihnen an die Bedingung der Anpassung von Therapien gekoppelt. Diese frühzeitige Begleitung durch eine medizinisch orientierte Fachexpertise hat unter anderem den Effekt, dass eine Schwangerschaft nicht intuitiv gelebt werden oder neue körperliche Erfahrungen vornehmlich mit nahestehenden Menschen kommuniziert werden können. Eine Folge ist auch, dass die ansonsten private Entscheidung für oder gegen ein Kind oder der Zeitpunkt einer Schwangerschaft nicht allein Sache des Paares sein können. Payne und McPhersons (2010) nennen die Schwangerschaft bei chronischer Erkrankung daher auch eine „public-private-experience“ [34]. Dies wird von einigen Frauen trotz der rationalen Anerkennung als Zumutung erlebt [34] [37] [39].

Viele der befragten Frauen erfahren, dass sie durch Ärzte bzw. Ärztinnen präventiv als Risikoschwangere mit der Folge engmaschiger und ausgeweiteter Diagnostiken und Therapien eingestuft werden. Nicht immer erscheint ihnen diese Sichtweise als angemessen. Sie konterkariert 
bereits zu Beginn der Schwangerschaft das Bedürfnis nach Normalität [33]. Einige Frauen beschreiben dies als ein „dem medizinischen System unterworfen sein“ [36] [37]. Das Risikoempfinden der Frauen und ihre Zuversicht bezüglich des zu erwartenden Schwangerschaftsverlaufs kann sich dabei wesentlich von dem der Ärzte bzw. Ärztinnen unterscheiden und ist auch von komplexen, teilweise biografischen Faktoren abhängig [2] [29]. Gleichzeitig sind die Ärzte bzw. Ärztinnen aber auch diejenigen, die ihnen bei der Umsetzung des Kinderwunsches helfen und als Ansprechpartner geschätzt sind, was aus Sicht einiger Frauen zu einem von Ambivalenz geprägten Betreuungsverhältnis führen kann [33]. In einigen Studien wird betont, dass sich Schwangere mit chronischer Erkrankung selten in Therapieentscheidungen integriert oder gefragt fühlen, was sie als sinnvolle Hilfe oder Unterstützung ansehen. Die Frauen vermuten, dass ihnen ein Urteil über das eigene Wohlergehen oder notwendige Behandlungen nicht zugetraut wird. Dies fördert unter anderen die Entwicklung unspezifischer Ängste und Befürchtungen [29] [36] [40].

Die Kontrolle über einzelne medizinische Aspekte der Schwangerschaft zu haben, wird als allgemeines Bedürfnis formuliert [33][36]. Dazu gehört, dass die Frauen über ihre Situation informiert sein wollen. Viele von ihnen beklagen jedoch, dass die verfügbaren Informationen nicht ihre spezifischen Problematiken berücksichtigen und Fragen daher unbeantwortet bleiben [29] [34] [35] [37] [40]. Payne und McPhersons [33] führen ergänzend an, dass die verfügbaren Informationen widersprüchlich sein können und damit möglicherweise zur Verwirrung und erhöhter Vulnerabilität führen. Zu viele Informationen können auch bewirken, dass die Frauen unter Entscheidungsdruck geraten, dem sie sich nicht gewachsen fühlen. Corbin (1987) empfiehlt, den Frauen ein Spektrum an Entscheidungsmöglichkeiten und Informationen anzubieten, um sie aktiv und fachlich begleitet in das Krankheitsmanagement einzubinden. Die Interviews mit den Frauen verdeutlichen, dass diese gerne kooperieren, wenn sie die Möglichkeit dazu bekommen und Vor-sowie Nachteile der Behandlungen kennen und abwägen können. Aufgrund der Komplexität der Fragestellungen ist dieses Ziel jedoch schwieriger zu erreichen als bei Frauen ohne Komplikationen und setzt hohe kommunikative Kompetenzen aller Beteiligten voraus.

Einige Forscher und Forscherinnen resümieren, dass eine gelungene Kommunikation zwischen der Schwangeren und den Akteuren des Gesundheitssystems eine große und oft ungenutzte Ressource darstellt. Da die oftmals komplexen Wechselwirkungen der Erkrankungen mit den jeweiligen Schwangerschaftsverläufen nur eingeschränkt standardisierbar sind und individuelle Entscheidungen erfordern, erhöht die Integration der Einschätzung der Schwangeren und ihrer Rückmeldung zu Symptomen und Befinden auch die Sicherheit von 
Mutter und Kind [2] [33]. Eine gelungene Integration der Perspektive der Frau in den Betreuungsprozess führt eher zur Inanspruchnahme und Akzeptanz medizinischer Leistungen [4]. Sie führt auch dazu, dass die Frauen trotz der oft weitreichenden Schwangerschaftsrisiken einen Effekt von Beruhigung und Freude bei der Inanspruchnahme von Vorsorgeangeboten schildern [2] [32].

Über Aspekte des Geburtserlebens werden in den Studien unterschiedliche Aussagen gemacht. Die Frauen beklagen eingeschränkte Wahlmöglichkeiten und die Nichtbeachtung ihrer Bedürfnisse bezüglich der Geburtsgestaltung [32] [35]. So weit möglich versuchen sie, sich vor unangenehmen Erfahrungen zu schützen. Dies tun sie unter anderem dadurch, dass sie vertraute Personen suchen, die ihre Interessen während der Geburt vertreten können. Viele Schwierigkeiten führen sie auf mangelnde Erfahrungen der Ärzte bzw. Ärztinnen und Hebammen mit ihrer Erkrankung zurück [29]. Im Krankenhaus erleben sie teilweise Unsicherheit darüber, in welcher Fachabteilung sie angemessen versorgt werden sollten. So schildern Frauen mit Rückenmarksverletzungen, dass sie nach der Geburt auf die Station für neurologische Erkrankungen statt auf die Wöchnerinnenstation verlegt wurden, was sie als diskriminierend erlebten [32].

\section{Das Kind im Fokus der mütterlichen Sorge}

In vielen Studien wird betont, dass sich die Frauen der Untersuchungsgruppen in gleichem Maße wie gesunde Frauen auf ein Kind freuen und die Schwangerschaft weit möglichst genießen [29] [33]. Die Schwangerschaft ist für Frauen mit chronischen Erkrankungen jedoch eine Zeit, in der sie neben der Freude auch Sorgen um die Gesundheit des Kindes aushalten müssen. Betroffene Frauen wissen, dass das Ungeborene, bedingt durch ihre eigene gesundheitliche Situation, bereits einen Patientenstatus innehat [37]. Oftmals wird die Angst geäußert, dem Kind die eigene Krankheit zu vererben oder es zu schädigen. Wurden die Frauen während einer medikamentösen Behandlung schwanger, sind diese Ängste besonders gravierend [34] [41].

Das Wohlergehen des Kindes hat in den Augen der Schwangeren Vorrang vor den eigenen Bedürfnissen und therapeutischen Notwendigkeiten. Bei allen den eigenen Körper betreffenden Therapieentscheidungen werden die Risiken und Vorteile für das Ungeborene mitbedacht [2] [33] [34] [37]. Das Wissen um die Wechselwirkung der Erkrankung mit dem kindlichen Wohlbefinden fördert einen ängstlich kontrollierenden Blick der Mutter auf das Ungeborene [2] [37]. Dies begünstigt einen dauerhaften Zustand von Stress, erhöhter Aufmerksamkeit und mütterlicher Sorge [33] [36].

\section{Umgang mit der Erkrankung in der Schwangerschaft}

Das Mutterwerden erleben viele der interviewten Frauen als eine Bestärkung ihrer Identität und ihres Frau seins. Diese Erfahrung kann dabei auch das langfristige Verhältnis zur Erkrankung verändern. Sie kann in den Hintergrund treten und einem Gefühl von gesellschaftlicher Teilnahme und Normalität weichen [29] [33] [37]. In der Phase der Schwangerschaft ist dieses Gefühl von Normalität nur eingeschränkt zu realisieren, weil mögliche mütterliche Beschwerden, Diagnosen und Therapien einen Sonderstatus innerhalb der Gruppe der Schwangeren nach sich ziehen und in einem Gefühl von „Ausgeschlossen-sein“ münden können [33] [36]. Da bekannte Strategien der Krankheitsbewältigung in der Schwangerschaft nicht mehr oder nur noch eingeschränkt wirksam sind, werden außerdem teils überwundene Ängste und Verunsicherungen reaktiviert [40].

Symptome der Schwangerschaft ähneln bisweilen diversen Krankheitssymptomen und können nicht immer von diesen unterschieden werden. Dies wird von den Frauen unterschiedlich bewertet. Bei Smeltzer (1994) deuten sie die Wahrnehmung von Schwangerschaftssymptomen wie Übelkeit als zusätzliche Verunsicherung [41]. Sie können aber auch als Beweis für Normalität und als positive Körpererfahrung jenseits von Krankheit interpretiert werden [33].

Nach Thomas [37] bedeuten die Erkrankung und die Schwangerschaft für die betroffenen Frauen zwei getrennte aber koexistierende „Karrieren“. „Karriere“ meint in diesem Fall die vielfältigen psychischen und sozialen Reaktionen und Anpassungsvorgänge, die der Patient im Verlauf der Erkrankung durchzustehen hat. Die Frauen bedenken je nach Erkrankung, dass sie während der Schwangerschaft gesundheitliche Risiken für den eigenen Körper und ihre langfristige relative Gesundheit eingehen [37] [41]. Die postpartale Zeit ist dadurch gekennzeichnet, dass die Frauen zum bewährten und mit relativer Autonomie gehandhabten Krankenmanagement zurückkehren wollen [34].

\section{Diskussion}

Die Phase der Schwangerschaft ist bei Frauen mit chronischer Erkrankung geprägt durch Stress und Anforderungen, die sich aus der gleichzeitigen Sorge um die eigene Gesundheit und die des Kindes sowie die kontinuierliche Auseinandersetzung mit medizinischen Therapien und Diagnostiken ergeben. Zumindest in der Ausprägung der körperlichen Veränderungen und der Dringlichkeit der Entscheidungsprozesse sind chronisch erkrankte Frauen in einer anderen Situation als Schwangere ohne Krankheitsdiagnose. Frauen mit chronischen Erkrankungen schildern, dass ihre Überlegungen von großer 
Unsicherheit durchdrungen sind und auch die Jahre nach der Geburt mit einbeziehen. Ein kontinuierliches Gefühl von Wachsamkeit und Aufmerksamkeit gegenüber den körperlichen Veränderungen geht mit diesem Fakt einher. Gerade wenn Krankheitsverläufe unberechenbar und im Verlauf nicht kalkulierbar sind, wird dies als beängstigend erlebt [36]. Eine zusätzliche Herausforderung für die Frauen ist, dass Informationen und Beratungsangebote zu Sexualität, Verhütung, Schwangerschaft, Geburt und Wochenbett auf gesunde Frauen abgestimmt sind [16] [20] [43] [44]. Dies erhöht das Gefühl, einen Sonderstatus innerhalb der Gruppe der Schwangeren einzunehmen. Dem allgemeinen Wunsch nach Normalität und Teilhabe steht dies entgegen.

Grundsätzlich werden die Sichtweisen und Bedürfnisse der Zielgruppe in den Konzepten der Geburtshilfe nur unzureichend berücksichtigt [17] [45].

\section{Fazit}

Die Erfahrungen der Frauen mit chronischer Erkrankung in der Zeit des Mutterwerdens sind neben der Freude von einer hohen Anspannung geprägt, die sich aus der Sorge um das Kind vor dem Hintergrund möglicher Komplikationen ergibt. Dies kann zu Konflikten bei Entscheidungen im Spannungsfeld eigener und kindlicher Gesundheit führen. Angebote und Informationen berücksichtigen selten ihre speziellen Bedürfnisse und Fragstellungen. Dem Wunsch nach Normalität steht gegenüber, dass betroffene Frauen einen Sonderstatus in der Gruppe der Schwangeren einnehmen.

Konzepte für die Betreuung der Frauen mit chronischer Erkrankung in Schwangerschaft, Geburt und Wochenbett sollten besser auf diese Gruppe abgestimmt werden. Es besteht Forschungsbedarf betreffs der Frage, inwieweit sich die Ergebnisse aufgrund der unterschiedlichen Versorgungssysteme auf Deutschland übertragen lassen. Hierzulande können Schwangere, Gebärende und Wöchnerinnen unabhängig vom Gesundheitsstatus zuzahlungsfrei und umfassend die Unterstützung von Ärzten bzw. Ärztinnen und Hebammen in Anspruch nehmen. In der Zusammenführung beider Berufskompetenzen könnte Potenzial für eine Versorgungsstruktur liegen, die betroffene Frauen bei der Abwägung von medizinischen Notwendigkeiten und individuellen Bedürfnissen unterstützt. Hebammen als auch im häuslichen Umfeld der Frau arbeitende Akteurinnen können helfen, die geforderte Integration der Familienmitglieder zu realisieren und in Abstimmung mit den betreuenden Ärzten bzw. Ärztinnen die medizinischen Erfordernisse auf die speziellen Bedürfnisse und Fragen der Frauen mit chronischer Erkrankung abzustimmen. 


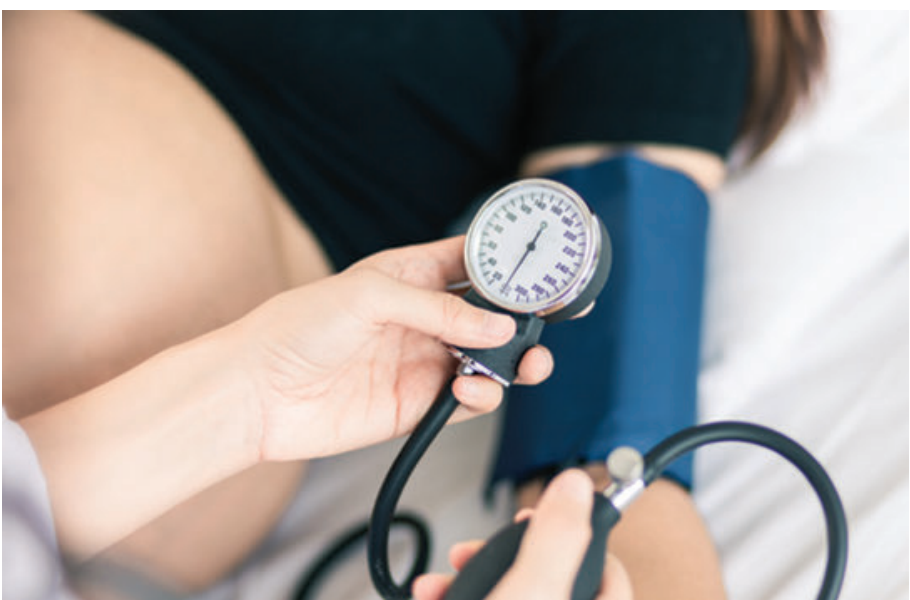

- Abb. 2 Schwangere Frauen mit chronischen Krankheiten können ihren Körper als potenzielle Bedrohung für ihr Kind wahrnehmen. (Foto: chompoo - stock.adobe.com, Symbolbild)

\section{Über diesen Artikel}

Dieser Beitrag basiert auf der Erstveröffentlichung, die in der Zeitschrift für Geburtshilfe und Neonatologie erschienen ist. Quelle: Lange U, Schnepp W, zu Sayn-Wittgenstein F. Das subjektive Erleben chronisch kranker Frauen in der Zeit von Schwangerschaft, Geburt und Wochenbett - eine Analyse qualitativer Studien. ZGN 2015; 219(04): 161-169. Der Beitrag war Teil eines Promotionsvorhabens mit Stipendium im Forschungsschwerpunkt Versorgung während Schwangerschaft, Geburt und Wochenbett - Instrumente zur sektorenübergreifenden Qualitätsentwicklung (IsQua) der Hochschule Osnabrück in Kooperation mit der Universität Witten / Herdecke, gefördert aus Landesmitteln des Niedersächsischen Ministeriums für Wissenschaft und Kultur.

\section{Autorinnen/Autoren}

Dr. rer. medic. Ute Lange ist Hebamme und Professorin für Hebammenwissenschaft am Department für Angewandte Gesundheitswissenschaften der Hochschule für Gesundheit Bochum. (Foto: hsg Bochum)

Dr. P. H. Friederike zu Sayn-Wittgenstein ist Hebamme und Professorin für Pflege- und Hebammenwissenschaft an der Hochschule Osnabrück

Korrespondenzadresse

Prof. Dr. Ute Lange

hsg Bochum | Hochschule für Gesundheit

Studienbereich Hebammenwissenschaft

Gesundheitscampus 6-8

44801 Bochum

E-Mail: ute.lange@hs-gesundheit.de

Literatur

[1] http://www.who.int/disabilities/world_report/2011/report. pdf World Report on Disability 2011. Genf. Online verfügbar unter zuletzt geprüft am 01.04.2014
[2] Scheidt-Nave C Chronische Erkrankungen - Epidemiologische Entwicklung und die Bedeutung für die Öffentliche Gesundheit. Public Health Forum 2010; 18: 2.e1-2.e3

[3] Gupton A, Heaman M, Cheung LW-K Complicated and Uncomplicated Pregnancies: Women's Perception of Risk. Journal of Obstetric, Gynecologic, \& Neonatal Nursing 2001; 30: 192-201

[4] Corbin J Women's perceptions and management of a pregnancy complicated by chronic illness. Health Care for Women International 1987; 8: 317-337

[5] Corbin J, Strauss A Weiterleben lernen. Verlauf und Bewältigung chronischer Krankheit. 2010; 3., überarb. Aufl.

[6] Dulude D, Bélanger C, Wright J High-risk pregnancies, psychological distress, and dyadic adjustment. Journal of Reproductive and Infant Psychology 2002; 20: 101-123 et al.

[7] Tuohy A, McVey C Experience of pregnancy and delivery as predictors of postpartum depression. Psychology, health \& medicine 2008; 13: 43-47

[8] Levy-Shiff R, Lerman M, Har-Even D Maternal adjustment and infant outcome in medically defined high-risk pregnancy. Developmental Psychology 2002; 38: 93-103 et al.

[9] http://ec.europa.eu/health/reports/docs/health_glance_en. pdf Health at a Glance: Europe 2010. 2002; Online verfügbar unter zuletzt geprüft am 18.10.2013

[10] Daten und Fakten: Ergebnisse der Studie „Gesundheit in Deutschland aktuell 2010“. Beiträge zur Gesundheitsberichterstattung des Bundes 2012. Hrsg

[11] Chatterjee S, Kotelchuck M, Sambamoorthi U Prevalence of Chronic Illness in Pregnancy, Access to Care, and Health Care Costs. Women's Health Issues 2008; 18: S107-S116

[12] Kersten I, Lange A, Haas J Chronic diseases in pregnant women: prevalence and birth outcomes based on the SNiPstudy. BMC Pregnancy and Childbirth 2014; 14 et al.

[13] https://www.sqg.de/downloads/Bundesauswertungen/2013/ bu_Gesamt_16N1-GEBH_2013.pdf Bundesauswertung zum Erfassungsjahr 2013. 16/1 Geburtshilfe Qualitätsindikatoren. Hrsg Online verfügbar unter zuletzt geprüft am 14.08.2014

[14] Malouf R, Redshaw M, Kurinczuk J Systematic review of heath care interventions to improve outcomes for women with disability and their family during pregnancy, birth and postnatal period. BMC Pregnancy and Childbirth 2014; 14 et al.

[15] Schaeffer D Bewältigung chronischer Erkrankung-Status Quo der Theoriediskussion. 2009; 15-51 1. Aufl

[16] Chuang C, Velott D, Weisman C Exploring Knowledge and Attitudes Related to Pregnancy and Preconception Health in Women with Chronic Medical Conditions. Maternal and Child Health Journal 2010; 14: 713-719

[17] Redshaw M, Malouf R, Gao H Women with disability: the experience of maternity care during pregnancy, labour and birth and the postnatal period. BMC Pregnancy Childbirth 2013; 174 et al.

[18] Prilleltensky O A Ramp to Motherhood: The Experiences of Mothers with Physical Disabilities. Sexuality and Disability 2013; 19: $21-47$

[19] Grue L, Lærum K ,Doing Motherhood': Some experiences of mothers with physical disabilities. Disability \& Society 2002; 17: 671-683

[20] Becker H, Stuifbergen A, Tinkle M Reproductive health care experiences of women with physical disabilities: a qualitative study. Archives of Physical Medicine \& Rehabilitation 1997; 78: $26-33$ 
[21] Stenhouse E, Letherby G, Stephen N Women with pre-existing diabetes and their experiences of maternity care services. Midwifery 2013; 29: 148-153

[22] Blegen N, Hummelvoll J, Severinsson E Experiences of motherhood when suffering from mental illness: A hermeneutic study. International Journal of Mental Health Nursing 2012; 21: 419-427

[23] Höglund B, Larsson M Struggling for motherhood with an intellectual disability - A qualitative study of women's experiences in Sweden. Midwifery 2013; 29: 698-704

[24] McConnell D, Mayes R, Llewellyn G Pre-partum distress in women with intellectual disabilities. Journal of Intellectual \& Developmental Disability 2008; 33: 177-183

[25] Lawrence J Women with diabetes in pregnancy: different perceptions and expectations. Best Practice \& Research Clinical Obstetrics \& Gynaecology 2011; 25: 15-24

[26] Crompton L Are HIV-infected pregnant women at a higher risk of developing depression?. British Journal of Midwifery 2013; 21: 204-213

[27] Wilson S 'When you have children, you're obliged to live': motherhood, chronic illness and biographical disruption. Sociology of Health \& Illness 2007; 29: 610-626

[28] Bagshaw J, Crawford P, Chappell B Problems that mothers' with epilepsy experience when caring for their children. Seizure 2008; 17: 42-48

[29] Walsh-Gallagher D, Sinclair M, Mc Conkey R The ambiguity of disabled women's experiences of pregnancy, childbirth and motherhood: A phenomenological understanding. Midwifery 2012; 28: 156-162

[30] http://www.caspinternational.org/?o=1012 CRITICAL APPRAISAL SKILLS PROGRAMME. Making sense of evidence about clinical effectiveness. 2006; Online verfügbar unter zuletzt geprüft am 16.09.2013

[31] Thomas J, Harden A Methods for the thematic synthesis of qualitative research in systematic reviews. BMC Medical Research Methodology 2008; 8: 45

[32] Tebbet M, Kennedy $P$ The experience of childbirth for women with spinal cord injuries: an interpretative phenomenology analysis study. Disability and Rehabilitation 2012; 34: 762-769

[33] Tyer-Viola L, Lopez R Pregnancy with Chronic Illness. Journal of Obstetric, Gynecologic, \& Neonatal Nursing 2014; 43: $25-37$
[34] Payne D, McPhersons K Becoming mothers. Multiple sclerosis and motherhood: A qualitative study. Disability and Rehabilitation 2010; 32: 629-638

[35] Lipson J, Rogers J Pregnancy, birth and disability: Women's health care experiences. Health Care for Women International 2000; 21: 11-26

[36] Berg M Pregnancy and Diabetes: How Women Handle the Challenges. Journal of Perinatal Education 2005; 14: 23-32

[37] Thomas H Pregnancy, illness and the concept of career. Sociology of Health and Illness 2003; 25: 383-407

[38] Smeltzer S The concerns of pregnant women with multiple sclerosis. Qualitative Health Research 1994; 4: 480-502

[39] Thomas C The baby and the bath water: disabled women and motherhood in social context. Sociology of Health and Illness 1997; 19: 622-643

[40] Thomas H Women's postnatal experience following a medically complicated pregnancy. Health Care for Women International 2004; 25: 76-87

[41] Smeltzer $S$ The concerns of pregnant women with multiple sclerosis. Qualitative Health Research 1994; 4: 480-502

[42] Franke A Modelle von Gesundheit und Krankheit. 2012; 3. überarb. Aufl

[43] Farber R Mothers With Disabilities: In Their Own Voice. American Journal of Occupational Therapy 2000; 54: 260-268

[44] Thomas C, Curtis P Having a baby: some disabled women's reproductive experiences. Midwifery 1997; 13: 200-209

[45] Meade T, Sharpe L, Hallab L Navigating Motherhood Choices in the context of Rheumatoid Arthritis: Women's Stories. Musculoskeletal Care 2013; 11: 73-82 et al.

[46] http://www.un.org/Depts/german/uebereinkommen/a r61106-dbgbl.pdf Teil II Nr. 35: Gesetz zum Übereinkommen der Vereinten Nationen über die Rechte von Menschen mit Behinderungen. 2008; Online verfügbar unter zuletzt geprüft am 21.06.2014

Bibliografie

DOI https://doi.org/10.1055/a-1084-5196

Die Hebamme 2020; 33: 40-47

(c) Georg Thieme Verlag KG Stuttgart · New York

ISSN 0932-8122 